\title{
A New Method for Studying He Damage in Materials Demonstrated on Nanotwinned Cu Nanopillars
}

\author{
Zhang-Jie Wang ${ }^{1,2}$, Frances Allen ${ }^{3}$, Zhi-Wei Shan ${ }^{2}$ and Peter Hosemann ${ }^{1}$ \\ 1. Department of Nuclear Engineering, University of California, Berkeley, California, 94720, USA \\ 2. Center for Advancing Materials Performance from the Nanoscale (CAMP-Nano) \& Hysitron Applied \\ Research Center in China (HARCC), State Key Laboratory for Mechanical Behavior of Materials, Xi'an \\ Jiaotong University, Xi'an, 710049, China \\ 3. Department of Materials Science and Engineering, University of California, Berkeley, California \\ 94720, USA
}

Ion implantation has been used for decades to investigate the response of materials to radiation damage. While displacement damage is caused due to an incoming particle, in high neutron energy environments gases are also produced due to transmutation or fission events. In particular fusion, fast reactor and spallation sources suffer from high He/dpa (displacements per atom) ratios. Understanding the effect of $\mathrm{He}$ in materials is a key aspect in these applications. It is known that interfaces are beneficial for radiation damage due to the fact that interfaces act as defect sinks. In the past He-denuded zones around grain boundaries have often been observed which prove that He can be managed by offering defect sinks [1]. Only a few studies have been performed investigating the effect of twin interfaces on $\mathrm{He}$ management [2]. The studies performed are rather limited in exploring different doses due to the fact that implanting the same grain with different doses and subsequent characterization of the resulting defects are challenging and rather time consuming, thus limiting systematic studies. Moreover, in order to obtain a direct comparison ideally one implants the same sample and same grain to different doses. Recently the Zeiss ORION NanoFab instrument was released which allows $\mathrm{He}$ and $\mathrm{Ne}$ ion beams in combination with a $\mathrm{Ga}$ ion source to quickly and efficiently manufacture nanostructures and direct $\mathrm{He}$ implantation [3]. In this work we utilize the combined Ga-He beam system to increase sample throughput and manufacture nanopillars with subsequent He implantation in a fast and efficient manner. Using this novel method one can manufacture nanopillars and implant these targeting the exact same grain in one session, thereby allowing for better and more accurate comparison and effect evaluation due to better controlled separate effect testing.

In this work several nanopillar samples were manufactured in one grain of a nanotwinned $\mathrm{Cu}$ sample. Each pillar was implanted to a different dose using $25 \mathrm{keV}$ He ions generated by the ORION NanoFab instrument. The maximum dose was $10^{19} \mathrm{He}^{+} / \mathrm{cm}^{2}$. Each pillar was then tested using a JEOL 2010 TEM equipped with a Hysitron PI95 nanomechanical testing system. Quantitative stress-strain data were collected in situ leading to a fundamental understanding of the deformation mechanism of the implanted pillars.

It was found that the approach of directly implanting $\mathrm{He}$ into nanotwinned $\mathrm{Cu}$ nanopillars can easily be conducted using the ORION NanoFab. Figures $1 \mathrm{a}$ and $\mathrm{b}$ show two nanotwinned pillars cut from one grain using a $\mathrm{Ga}$ ion source and then implanted with different doses of He. In the case of samples implanted with a high dose (e.g. $10^{18} \mathrm{He}+/ \mathrm{cm}^{2}$ ) He bubbles were formed in the material, as shown in the defocused bright field image in Figure 1c. Micro compression testing leads to the stress-strain curve shown in Figure 2. The strength of the nanopillars significantly depends on the dose of He. 
Taking advantage of the accurate and controllable micro area He implantation technique described, different doses of $\mathrm{He}$ ions were implanted into $\mathrm{Cu}$ nanotwinned pillars manufactured in one grain. The influence of various $\mathrm{He}$ doses on the mechanical properties of nanotwinned $\mathrm{Cu}$ was systematically studied by in situ mechanical testing. This novel technique makes it feasible to evaluate He ion damage and its effect on small volume materials.

\section{References:}

[1] M. Zhernenkov et al., Trapping of implanted $\mathrm{He}$ at $\mathrm{Cu} / \mathrm{Nb}$ interfaces measured by neutron reflectometry. Appl. Phys. Lett. 98, (2011), 241913.

[2] K. Yu et al., Removal of stacking-fault tetrahedra by twin boundaries in nanotwinned metals. Nature Communications 4, (2013), 1377.

[3] V. Veligura et al., Digging gold: keV He+ion interaction with Au, Beilstein J. Nanotechnol.,4, (2013), 453-460.

[4] The Authors thank The China Scholarship Council for providing funding for the visiting scientist. This publication was made possible in part by NSF/DMR MRI DMR-1338139. This research is performed using funding received from the DOE Office of Nuclear Energy's Nuclear Energy University Programs and the Keck Foundation. The nanotwinned pillars were prepared and implanted at the Biomolecular Nanotechnology Center at the University of California, Berkeley, and the TEM in situ analysis was performed at The Molecular Foundry, which is supported by the Office of Science, Office of Basic Energy Sciences, of the U.S. Department of Energy under Contract No. DE-AC02-05CH11231.
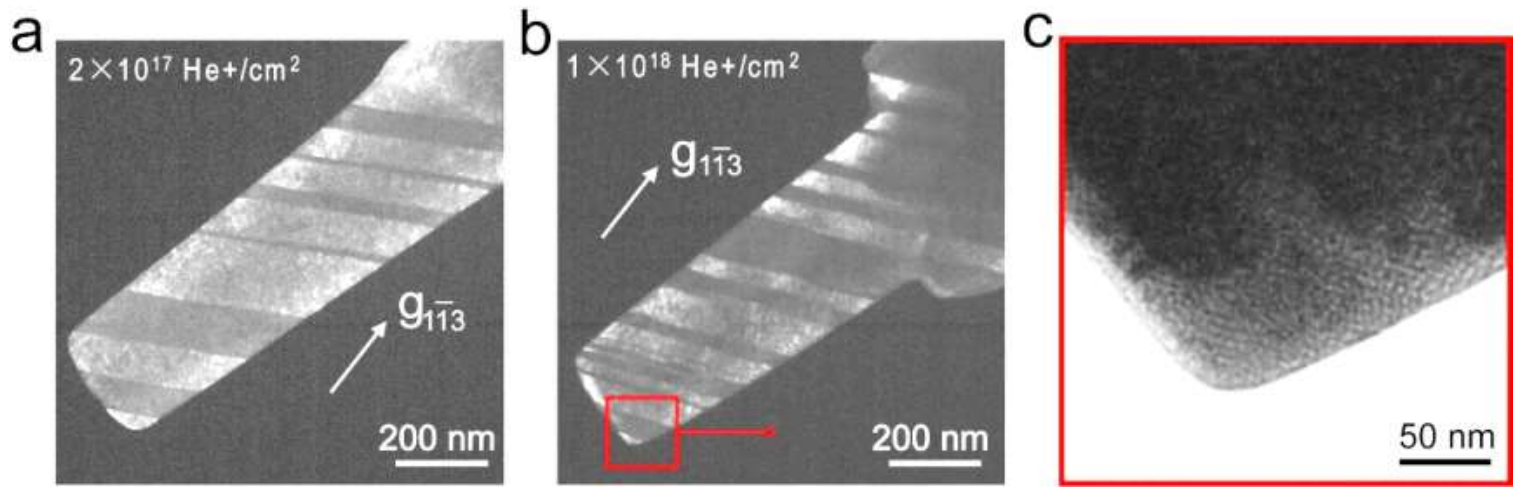

Figure 1 nanotwinned pillars with different doses of He.

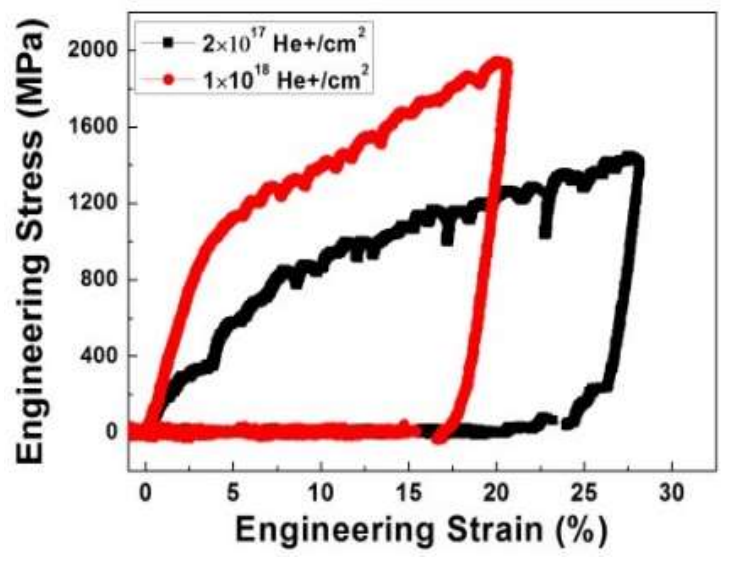

Figure 2 Engineering stress versus engineering strain curves of nanotwinned $\mathrm{Cu}$ nanopillars implanted with two different doses of He. 